thresholds was selected based on multiple considerations including clinical validity, optimization of sensitivity and specificity, and pragmatism.

Results. A total of 160 clinical interviews were conducted; 139 patients had clinical interview-confirmed BPD-I $(n=67)$ or MDD ( $n=72)$. The screening tool was reduced from 10 to 6 items based on item-level analysis. When 4 items or more were endorsed (yes) in this analysis sample, the sensitivity of this tool for identifying patients with BPD-I was 0.88 and specificity was 0.80 ; positive and negative predictive values were 0.80 and 0.88 , respectively. These properties represent an improvement over the Mood Disorder Questionnaire, while using $>50 \%$ fewer items.

Conclusion. This new 6-item BPD-I screening tool serves to differentiate BPD-I from MDD in patients with depressive symptoms. Use of this tool can provide real-world guidance to primary care practitioners on whether more comprehensive assessment for BPD-I is warranted. Use of a brief and valid tool provides an opportunity to reduce misdiagnosis, improve treatment selection, and enhance health outcomes in busy clinical practices.

Funding. AbbVie Inc.

\section{Psychiatry on a Shoestring: Developing New Standards of Care for a Severe, Prolonged, and Widespread Emergency}

\section{James E. Black, MD, PhD, MPH}

University of Minnesota Medical School, Department of Psychiatry, St. Paul, MN, USA

Presenting Author: James E. Black

\section{Abstract}

Study Objective. The COVID-19 crisis has severely stressed our healthcare system and pushed our economy to the brink. This long emergency will probably cause years of severe suffering in every region. Health expenses greatly increased, supply chains were disrupted, and governments coped with much less revenue. Good clinicians plan for ALL contingencies, and we need to consider that the current disaster may get much worse. How can we adapt psychiatry to a long emergency? This goes far beyond previous work on crisis standards of care because the emergency is severe, prolonged, and widespread. If we had to spend much less on psychotropics, which meds stay on the formulary? If we have to close hospitals, which patients get a bed? What adaptations could be used if demand exceeds the supply of providers? Very little is known about how to make severe, permanent cuts to healthcare. Our previous systematic review found no scholarship addressing the ethics of severe and prolonged healthcare rationing. Global catastrophes need a global health policy, but this one has no experts. The present study starts the project by surveying experts with related experience that could be useful in future plans.
Method. We used purposive sampling to find 18 professionals with experience in healthcare rationing from underserved, indigenous communities, homeless programs, and African nations. We also interviewed ethicists, pharmacists, administrators, NGO clinicians, and military. Interviews were transcribed and coded using basic inductive techniques. Because so little is known about this topic, we used grounded theory, an iterative approach to guide further sampling, refine interviews, and make some preliminary conclusions.

Results. Participants all agreed this crisis planning is extremely important and complex. They described diverse concerns regarding ethical decision making, with some having confidence with top-down government policy, and others recommending a grassroots approach. Minority participants had less confidence in government. There was no consensus on any best ethical framework. Most had confidence that clinicians will ultimately do the right thing. Native American leaders had confidence in a holistic, preventive approach. All agreed that social justice should be central in measuring economic impact of long emergencies and choosing ethical options. We collected suggestions for innovative approaches to rationing.

Conclusions. This research program illuminates the difficult ethical questions about adapting psychiatry to a prolonged, widespread, and severe emergency. Our interviews identify areas where severe but ethical cuts can be made in medications, hospitals, clinical staff, and administration. Next steps include evidence-based formularies, utilitarian staff cuts, and ethical standards for closing beds or revamping state hospitals. Underserved and diverse communities with rationing experience must have a voice in the discussion.

\title{
The Incidence and Economic Burden of Extrapyramidal Symptoms in Patients with Schizophrenia Treated with Atypical Antipsychotics
}

Aditi Kadakia, $\mathrm{MS}^{1}$, Brenna L. Brady, $\mathrm{PhD}^{2}$, Huan Huang, $\mathrm{PhD}^{1}$, Carole Dembek, $\mathrm{MS}^{1}$, G. Rhys Williams, DSc $^{1}$ and Justine M. Kent, MD ${ }^{1}$

${ }^{1}$ Sunovion Pharmaceuticals Inc., Marlborough, MA, USA, and ${ }^{2}$ IBM Watson Health, Bethesda, MD, USA

Presenting Author: Aditi Kadakia 
ICD-10 diagnoses and medications) was assessed during the 12-months following AAP initiation. Cohorts with and without EPS were defined. Demographics, clinical characteristics, and healthcare resource use and costs over 12 months following the first EPS claim (EPS) or randomly assigned index date (Non-EPS) were assessed.

Results. A total of 11,642 patients with schizophrenia were identified; $21.2 \%$ developed EPS in the 12-months following AAP initiation. EPS and Non-EPS cohorts included 2,295 (mean age $38,61 \%$ male, CCI 0.6 ) and 5,607 (mean age $39,57 \%$ male, CCI 0.7) patients, respectively. Over the 12 -month post-index period, EPS cohort had significantly higher rates of all-cause (30.2\% vs. $24.6 \%, \mathrm{p}<0.001)$ and schizophrenia-related hospitalizations $(22.5 \%$ vs. $12.9 \%, \mathrm{p}<0.001)$ and schizophrenia-related emergency room visits $(25.5 \%$ vs. $16.7 \%, \mathrm{p}<0.001)$ compared to Non-EPS cohort. All-cause $(\$ 25,911$ vs. $\$ 21,550, \mathrm{p}<0.001)$ and schizophrenia-related healthcare costs $(\$ 12,134$ vs. $\$ 6,230$, $\mathrm{p}<0.001)$ were significantly higher in EPS vs. Non-EPS cohort. Conclusions. In the 12 months following AAP initiation, over $20 \%$ of schizophrenia patients developed EPS, which was associated with increased healthcare resource utilization and costs. Treatment options that minimize EPS may reduce the economic burden of schizophrenia.

Funding. Sunovion Pharmaceuticals Inc.

\section{Is this Withdrawal or Intoxication? Case Report Regarding Complications of Unregulated Use of Tianeptine, Etizolam, and Phenibut in the USA}

\section{Sadia B. Ghani, MD, Eric Taylor, MD and \\ Siddesh Gopalakrishnan, MD}

University of Arizona, Department of Psychiatry, Tucson, AZ, USA

Presenting Author: Sadia B. Ghani, Eric Taylor, Siddesh Gopalakrishnan

\section{Abstract}

Background. The internet allows easy access for the sales of psychoactive agents that are not regulated by the FDA. Some of those agents are used to help manage anxiety, depression and sleep, such as tianeptine, etizolam, and phenibut. These medications have the potential for abuse and potentially leading to altered mental status when intoxicated or withdrawing. This presents a challenge to clinicians who may not be aware of availability of such substances. Available literature has discussed the use of above substances individually, but how do you treat if there is use of more than one substance with different mechanisms of actions? Here we present a case of an adult male who has used all three agents simultaneously, leading to a hospital admission.
\end{abstract}

Case History. A 32-year-old male presented to the emergency department (ED) for altered mental status (AMS). He has a documented history of anxiety but was never treated with prescription medications. No history of substance use was documented. He was self-medicating with concurrent use of tianeptine (atypical antidepressant with mu agonist properties,) phenibut (GABA mimetic) and etizolam (a benzodiazepine-like agent). During his stay, he was agitated and delirious with reports of visual hallucinations. Neuroimaging and lab studies were within normal limits, EEG showed no seizure activity. Over the course of his hospital stay, he was started on Depakote for agitation, a Valium taper for suspected benzodiazepine withdrawal and prevention of seizures, Seroquel for delirium, and baclofen for suspected GABAergic withdrawal symptoms. The patient's AMS improved and he was discharged on hospital day 10.

Conclusions. This case illustrates the difficulty managing poly-substance use/abuse and stresses the importance for physicians to screen for psychoactive agents purchased over the internet or over the counter to improve treatment outcomes. Continued discussions with patients regarding risks/ benefits of use of such substances would be beneficial and help increase awareness.

\section{Safety of Using a Combinatorial Pharmacogenomic Test for Patients with Major Depressive Disorder in the GUIDED trial}

\author{
Sagar V. Parikh, MD' ${ }^{1}$, Gabriela K. Khazanov, $\mathrm{PhD}^{2}$, \\ Michael E. Thase, $M^{2}$, Anthony J. Rothschild, $M^{3}{ }^{3}$, \\ Boadie W. Dunlop, $\mathrm{MD}^{4}$, Charles DeBattista, $\mathrm{DMH}, \mathrm{MD}^{5}$, \\ Charles R. Conway, $\mathrm{MD}^{6}$, Brent P. Forester, $\mathrm{MD}, \mathrm{MSc}^{7}$, \\ Richard C. Shelton, $\mathrm{MD}^{8}$, Matthew Macaluso, $\mathrm{DO}^{9}$, \\ James Li, PhD ${ }^{10}$, Kunbo Yu, MS ${ }^{10}$, \\ Michael R. Jablonski, $\mathrm{PhD}^{10}$, Stephanie Meek, $\mathrm{PhD}^{11}$ and \\ John F. Greden, MD ${ }^{1}$
}

\footnotetext{
${ }^{1}$ University of Michigan, Comprehensive Depression Center and Department of Psychiatry, and National Network of Depression Centers, Ann Arbor, MI, USA, ${ }^{2}$ University of Pennsylvania, Perelman School of Medicine, and the Corporal Michael Crescenz VAMC, Philadelphia, PA, USA, ${ }^{3}$ University of Massachusetts Medical School and UMass Memorial Healthcare, Worcester, MA, USA, ${ }^{4}$ Emory University School of Medicine, Department of Psychiatry and Behavioral Sciences, Atlanta, GA, USA, ${ }^{5}$ Stanford University School of Medicine, Stanford, CA, USA, ${ }^{6}$ Washington University School of Medicine, St. Louis, MO, USA, ${ }^{7}$ McLean Hospital, Belmont, MA, USA, ${ }^{8}$ The University of Alabama at Birmingham, Birmingham, AL, USA, ${ }^{9}$ University of Kansas School of Medicine-Wichita, Wichita, KS, USA, ${ }^{10}$ Myriad Neuroscience, Mason, OH, USA, and ${ }^{11}$ Myriad Genetics, Inc., Salt Lake City, UT, USA
}

Presenting Author: Sagar V. Parikh 